\title{
On the Nose: Anti-MDA-5 Dermatomyositis Manifesting as Perinasal Swelling
}

\author{
Emily Molina $^{a} \quad$ Lisa Christopher-Stine $^{b} \quad$ Jemima Albayda ${ }^{b}$ \\ aDepartment of Internal Medicine, Johns Hopkins University School of Medicine, Baltimore, \\ MD, USA; b Division of Rheumatology, Johns Hopkins University School of Medicine, \\ Baltimore, MD, USA
}

\section{Keywords}

Dermatomyositis · Anti-MDA-5 antibody · Cutaneous manifestations · Myositis-specific antibodies

\begin{abstract}
The clinical presentation of dermatomyositis (DM) is diverse, with varied phenotypes that may be correlated with specific autoantibodies. The anti-melanoma differentiation-associated gene 5 (MDA5) antibody in DM is associated with an amyopathic phenotype of DM, with several unusual cutaneous manifestation and increased risk for rapidly progressive interstitial lung disease. Initial presentation may be subtle, but early diagnosis is key to initiation of proper immunosuppressive therapy. In this report, we describe perinasal edema and erythema as a presenting complaint of anti-MDA5 DM in an otherwise healthy 40-year-old woman. The edema began shortly after heavy sun exposure and was followed by painful papules in her hands and arthritis within a few weeks. She was found to have high titer of anti-CCP and antiMDA5, and thus was diagnosed with DM and rheumatoid arthritis overlap. A CT chest, abdomen, and pelvis showed patchy ground-glass and interstitial opacities in bilateral lower lobes consistent with mild interstitial lung disease without evidence of malignancy. Perinasal cutaneous findings and arthralgias improved with initiation of prednisone. To our knowledge, this is the first report of perinasal edema as a presenting symptom for DM and should raise suspicion for MDA-5 disease.
\end{abstract}




\section{Introduction}

The clinical presentation of dermatomyositis (DM) is diverse, with varied phenotypes that may be correlated with specific autoantibodies [1]. The anti-melanoma differentiationassociated gene 5 (MDA5) antibody in DM is associated with a lower incidence of myositis with an increased risk for rapidly progressive interstitial lung disease [2]. Here, we describe a patient with perinasal edema as a presenting complaint in MDA-5 DM to raise awareness of unique and distinctive cutaneous features in this enigmatic disease.

\section{Case Report/Case Presentation}

A 40-year-old Caucasian woman with no significant past medical history presented with a 1-month history of painless swelling around the dorsum and nasal sidewall which began within days of a trip to the beach with heavy sun exposure (shown in Fig. 1a). This was soon followed by an erythematous rash across the cheeks and nasolabial folds. She was evaluated by an otolaryngologist and was treated with antibiotics for presumed sinusitis without improvement. Routine CBC and CMP, and a CT of the sinuses were unremarkable. A few weeks later, she developed joint stiffness and swelling of the hands and feet. This was accompanied by erythematous and painful papules at the palmar aspect of her fingers (shown in Fig. 1b), Gottron's papules (shown in Fig. 1c), and an erythematous rash across her chest. Further workup revealed an ANA 1:320, and a high-titer anti-CCP and anti-RF. A myositis panel showed a positive anti-MDA5 antibody at high titer. She was started on prednisone $10 \mathrm{mg}$ daily with immediate improvement in her perinasal swelling and cutaneous findings. A CT chest showed patchy ground-glass and interstitial opacities in bilateral lower lobes consistent with mild interstitial lung disease and pulmonary function tests were normal. There was no evidence of malignancy in an abdominal/pelvic CT. She had normal muscle enzymes and no subjective or objective weakness. She was diagnosed with MDA-5 DM with an overlap of rheumatoid arthritis, and was started on methotrexate and higher doses of steroids. Given persistence of palmar rashes and joint swelling, she was eventually transitioned to Rituximab which led to disease control.

\section{Discussion/Conclusion}

Patients with anti-MDA5 DM represent a distinct subtype of DM, with several unique cutaneous features differing considerably from the classic ones of DM, such as cutaneous and oral ulcerations, palmar papules, alopecia, and panniculitis [3]. The list of unusual manifestations of this syndrome continues to grow, including descriptions of unilateral heliotrope rash and eyelid swelling as a first sign of this phenotype [4,5]. In a case series of 3 patients described by Kume et al. [6], they also found that this unilateral heliotrope rash was only seen in DM patients with MDA-5 antibodies, preceded the usual manifestations of DM, and could be associated with rapidly progressive interstitial lung disease. Another unique early finding that has been reported in MDA-5 DM is lobular panniculitis of the cheek or mandible [7, 8]. In both cases, swelling and unilateral erythema of the angle of the jaw was seen, and correlated with a lobular panniculitis of lymphoplasma-histiocytic infiltrates that rapidly improved with treatment. More typical skin findings of DM as well as pulmonary involvement followed these initial eruptions.

The exact mechanisms underlying the edematous cutaneous manifestations seen in DM are unclear. Increased dermal mucin has been described together with an interface dermatitis in periorbital edema [9]. Interestingly, there has been growing evidence that anti-MDA5

\section{Karger'}




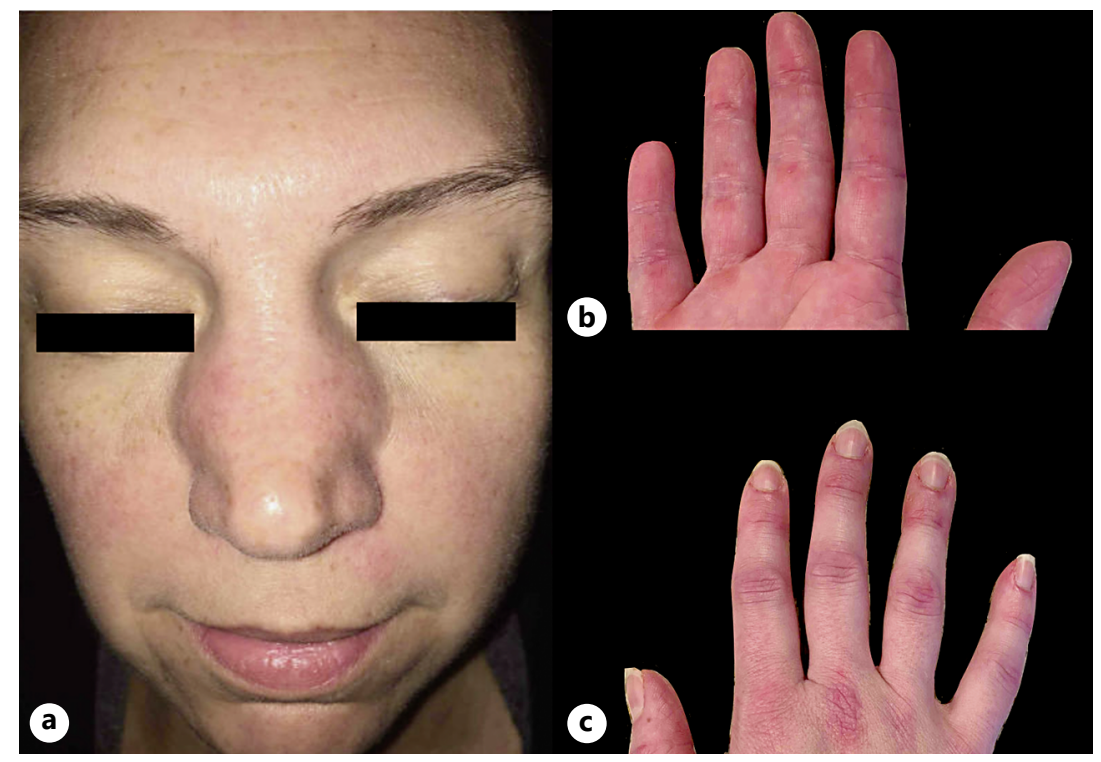

Fig. 1. a Perinasal edema and mild erythema on bilateral cheeks. b Palmar surface of the right-hand showing painful papules described in MDA-5 DM. c Dorsal surface of the right hand showing violaceous rash on the surface of the joints consistent with Gottron's papules.

affects the type 1 interferon (T1-IFN) pathway [10], which has been associated with more extensive skin involvement, including palmar papules and cutaneous ulcerations [3]. MDA-5 is a key viral sensor [11] and activating this pathway is a common antiviral response in healthy skin presenting as dermatitis [12]. T1-IFNs are known to have vasculopathic effects [13], which raises the question of whether endothelial injury may be driving both the cutaneous manifestations and interstitial lung remodeling associated with this subset of DM. In fact, a recent study showed that anti-MDA5 patients had high titers of T1-IFN in serum and affected skin [14].

To our knowledge, this is the first case to describe perinasal swelling in DM and may indicate a spectrum of facial or cutaneous involvement particular to MDA-5 disease. The differential diagnosis for isolated perinasal swelling would be quite limited and may include allergic and local reactions, granulomatous disease, T-cell lymphoma, cellulitis, or rosacea. However, a history of cutaneous changes presenting days after heavy sun exposure should also raise suspicion for an autoimmune process. While UV-exposure is more commonly associated with triggering cutaneous features of systemic lupus erythematosus, it is also implicated in DM [15]. Additionally, while the manifestation has never before been described in $\mathrm{DM}$, the development of more typical DM rashes later on in the course of her disease strengthens this association. This case highlights a novel and unique finding of anti-MDA5 DM that if recognized, may lead to an earlier diagnosis and improved outcome for patients with this rare disease.

\section{Statement of Ethics}

Written informed consent was obtained from the patient for publication of the details of this medical case and any accompanying images. The case complied with the Declaration of Helsinki. Ethical approval beyond written informed consent that was obtained was not required for this study in accordance with local/national guidelines. 


\section{Conflict of Interest Statement}

The authors have no conflicts of interest to disclose.

\section{Funding Sources}

This work was supported by the Jerome L. Greene Foundation and Cupid Foundation to J.A.

\section{Author Contributions}

J.A. was involved in contact with the patient, obtaining written consent, and diagnosis of the patient. E.M., J.A., and L.C.S. contributed to literature review, manuscript writing, and reviewing.

\section{Data Availability Statement}

All data are available in this manuscript.

\section{References}

1 McHugh NJ, Targoff IN. Role of ANA and myositis autoantibodies in diagnosis. In: Aggarwal R, Oddis CV, editors. Managing myositis. Springer International Publishing; 2020. p. 167-74.

2 Kurtzman DJB, Vleugels RA, Tucson B, Arizona B. Anti-melanoma differentiationeassociated gene 5 (MDA5) dermatomyositis: a concise review with an emphasis on distinctive clinical features. J Am Acad Dermatol. 2018 Apr;78(4):776-85.

3 Fiorentino D, Chung L, Zwerner J, Rosen A, Casciola-Rosen L. The mucocutaneous and systemic phenotype of dermatomyositis patients with antibodies to MDA5 (CADM-140): a retrospective study. J Am Acad Dermatol. 2011;65(1):25-34.

4 Lam SC, Yuen HKL. Unilateral eyelid swelling as a sign of antimelanoma differentiation-associated gene 5 (Anti-MDA5)-antibody-positive dermatomyositis. Ophthalmic Plast Reconstr Surg. 2018;34(6):e209-11.

5 Al-Janobi G, Alkhalidi H, Omair MA. Unilateral heliotrope rash in juvenile dermatomyositis: an unusual presentation of an underlying serious disease. Case Rep Rheumatol. 2014;2014:979856-3.

6 Kume M, Arase N, Okiyama N, Koguchi-Yoshioka H, Tada T, Saruban H, et al. Unilateral heliotrope rash: a warning sign for anti-melanoma differentiation-associated gene 5 antibody-positive dermatomyositis. Rheumatology. 2021;60(4):e134-5.

7 Hattori Y, Matsuyama K, Takahashi T, Shu E, Kanoh H, Seishima M. Anti-MDA5 antibody-positive dermatomyositis presenting with cellulitis-like erythema on the mandible as an initial symptom. Case Rep Dermatol. 2018;10(2):110-4.

8 Galli S, Naudi G, Guler SA, Genitsch V, Cullmann J, Stefanski AL, et al. Kissed by MDA-5: lobular panniculitis of the cheek as an initial symptom of dermatomyositis. Rheumatology. 2020;59(5):1189.

9 Sevigny GM, Mathes BM. Periorbital edema as the presenting sign of juvenile dermatomyositis. Pediatr Dermatol. 1999;16(1):43-5.

10 Melki I, Devilliers H, Gitiaux C, Bondet V, Duffy D, Charuel JL, et al. Anti-MDA5 juvenile idiopathic inflammatory myopathy: a specific subgroup defined by differentially enhanced interferon- $\alpha$ signalling. Rheumatology. 2020;59(8):1927-37.

11 Fedorov M. Pandemic of autoimmunity: SARS-CoV-2 antibodies as autoantibodies causing ILD alike atypical pneumonia Target designation theory of immune pathology and autoimmunity view project pandemic of autoimmunity: SARS-CoV-2 antibodies as autoantibodies causing IL; 2020.

12 Hile GA, Gudjonsson JE, Kahlenberg JM. The influence of interferon on healthy and diseased skin. Cytokine. 2020;132:154605.

13 Feldman D, Goldstein AL, Cox DC, Grimley PM. Cultured human endothelial cells treated with recombinant leukocyte A interferon. Tubuloreticular inclusion formation, antiproliferative effect, and $2^{\prime}-5^{\prime}$ oligoadenylate synthetase induction. Lab Investig. 1988;58(5):584-9. 
14 Ono N, Kai K, Maruyama A, Sakai M, Sadanaga Y, Koarada S, et al. The relationship between type 1 IFN and vasculopathy in anti-MDA5 antibody-positive dermatomyositis patients. Rheumatology. 2019;58(5):78691.

15 Bogdanov I, Kazandjieva J, Darlenski R, Tsankov N. Dermatomyositis: current concepts. Clin Dermatol. 2018; 36(4):450-8. 